'Departamento de Laboratorios Clínicos, Facultad de Medicina, Pontificia Universidad Católica de Chile. Santiago, Chile.

aResidente de Laboratorio Clínico. bIngeniero en Computación, PhD en Ingeniería Mención Computación

*David Rodríguez y Elena Nieto comparten contribuciones para primera autoría del artículo.

El trabajo no recibió financiamiento. Los autores declaran no tener conflictos de interés.

Recibido el 3 de abril de 2020, aceptado el 7 de septiembre de 2020.

Correspondencia a:

Dr. Francisco Vera Vicuña Mackenna 4686, Macul. Santiago, Chile. fjvera1@uc.cl

\section{Eficacia en el control de la anticoagulación de los diferentes fármacos antagonistas de la vitamina $K$ disponibles en Chile}

\author{
DAVID RODRÍGUEZ ${ }^{1, \mathrm{a}}$, ELENA NIETO ${ }^{1}$, TERESA QUIROGA $^{1}$, \\ ERIC ROJAS $^{1, \mathrm{~b}}$, FRANCISCO VERA $^{1, \mathrm{a}}$
}

\section{Anticoagulant efficacy of different pharmacological presentations of vitamin $\mathrm{K}$ antagonists}

Background: Vitamin Kantagonist medications (VKA) are essential for the prevention of thromboembolic events, but their effectiveness is influenced by multiple factors, such as the type of medication chosen. Aim: To evaluate the efficacy in anticoagulant control of the bioequivalent and non-bioequivalent drugs of acenocoumarol compared to the reference drug. To evaluate the efficacy of warfarin bioequivalents available in Chile. To contrast the overall anticoagulant control efficacy between acenocoumarol and warfarin. Material and Methods: The results of 69333 outpatient oral anticoagulation controls were analyzed. Patient were separated in groups according to the drug that they used. Subsequently, the proportions of controls outside the range for each of acenocoumarol and warfarin bioequivalent drugs were compared. Acenocoumarol non-bioequivalent drugs were also compared with the reference drug. Acenocoumarol was compared with warfarin. Results: Acenocoumarol bioequivalent drugs and the reference drug had a similar proportion of controls outside the range (Odds ratios (OR) 0.812; 0.969; 0.974 and 0.963). Non-bioequivalent drugs had a higher proportion than the reference drug (OR 1.561 and 2.037). Both warfarin brands have a similar proportion of controls outside of the range (OR 1.050). Acenocoumarol compared to warfarin had a significant higher proportion of controls outside the range (OR 1.191). Conclusions: The pharmacological presentation of vitamin $K$ antagonists could influence anticoagulant control. Therefore, it is not prudent to switch these presentations frequently.

(Rev Med Chile 2020; 148: 1254-1260)

Key words: Acenocoumarol; Anticoagulants; Therapeutic Equivalency; Warfarin.
E $\mathrm{n}$ la actualidad, el uso de fármacos anticoagulantes orales constituye una herramienta esencial para la prevención primaria y secundaria de eventos tromboembólicos en diversas situaciones clínicas, entre las cuales destacan la fibrilación auricular, las afecciones valvulares del corazón y el tromboembolismo venoso ${ }^{1}$. Dentro de los fármacos disponibles para este efecto, los más utilizados son los antagonistas de la vitamina K (AVK). En Chile se encuentran disponibles dos principios activos de AVK: acenocumarol y warfarina.

La enfermedad cardiovascular (ECV) es la principal causa de muerte en todo el mundo; en 
Chile, en el año 2012 la tasa general de mortalidad atribuible a las ECV fue de 156,2 por 100.000 habitantes 160,2 para hombres y 152,2 para mujeres ${ }^{2}$. Los últimos datos publicados por el Departamento de Estadística e Información de Salud de Chile, para 2017, establecen que el número de visitas a los servicios de emergencia supera los 6.000 casos por semana para pacientes con ECV. En muchos pacientes que sobreviven a una enfermedad cardiovascular se prescribe un anticoagulante oral para prevenir futuros eventos tromboembólicos ${ }^{1}$, en especial AVK.

El tratamiento con AVK debe ser supervisado estrechamente ya que la eficacia y la seguridad de estos fármacos dependen del mantenimiento del efecto anticoagulante dentro de un margen terapéutico definido y estrecho ${ }^{3,4}$. La acción de estos medicamentos es monitorizada utilizando el tiempo de protrombina, a través de una fórmula de estandarización denominada International Normalized Ratio (INR). Tener un INR sobre o bajo los niveles terapéuticos para la indicación específica, expone al paciente a un mayor riesgo de presentar complicaciones hemorrágicas o tromboembólicas, respectivamente. Por ello, el manejo de la terapia anticoagulante oral requiere ajustes precisos de dosis, pero la respuesta de un individuo a los cumarínicos depende de varios factores ${ }^{5,6}:$ a) los factores no genéticos que incluyen la edad, el sexo, el índice de masa corporal, la dieta y los medicamentos que el paciente utiliza que tienen interacciones potenciales que aumentan o disminuyen el efecto anticoagulante ${ }^{7}$; b) los factores genéticos ${ }^{6,8,9}$ dentro de los que se encuentra la función del sistema citocromo P450 y la subunidad del complejo de vitamina K epóxido reductasa que tienen un papel clave en el metabolismo de estos medicamentos (genes codificantes de las enzimas que metabolizan el fármaco y la vitamina K). En la práctica clínica habitual, mantener un nivel de anticoagulación estable es difícil. En el mejor de los casos, en centros con programas de anticoagulación bien estructurados y rigurosos, se logra que los pacientes permanezcan con INR en el rango terapéutico asignado según su patología definido como tiempo en rango terapéutico $(\mathrm{TRT})^{10}$, durante al menos $65 \%$, lo que se traduce en tasas de accidente cerebrovascular/embolia sistémica y hemorragia más bajas así como en una menor mortalidad ${ }^{11}$. El National Institute for Health and Care Excellence (NICE) recomienda que el TRT debe ser superior a $65 \%{ }^{12}$, la Sociedad Europea de Cardiología recomienda un TRT objetivo de al menos $70 \%{ }^{13}$, y la Asia Pacific Heart Rhythm Society recomienda un TRT de al menos $60 \%$ para un control óptimo de $\mathrm{AVK}^{14}$.

Es conocido que el fármaco elegido (principio activo) podría tener un rol clave en el adecuado control anticoagulante ${ }^{15,16}$, y se desconoce cuál es la presentación de los principios activos de AVK (de los disponibles en Chile) que tiene una tasa de mayor de éxito en el control de anticoagulación respecto al fármaco considerado de patente inicial (fármaco de referencia), por lo que se formulan los siguientes objetivos: a) evaluar la eficacia en el control anticoagulante de los fármacos bioequivalentes del acenocumarol y warfarina en Chile en comparación al fármaco de referencia; b) evaluar la eficacia en el control anticoagulante de los fármacos no registrados como bioequivalentes del acenocumarol en Chile en comparación al fármaco de referencia y c) contrastar la eficacia global en el control anticoagulante entre el acenocumarol y la warfarina.

\section{Material y Método}

Se realizó un estudio de tipo transversal, en el cual se recopilaron los resultados de los controles de pacientes ambulatorios realizados en el policlínico de control de terapia anticoagulante oral con AVK (TACO) de la Red de Salud UC-CHRISTUS contenidos en el sistema informático del servicio entre enero de 2018 y diciembre de 2019 $(\mathrm{n}=69.322)$ realizados por 6 médicos especialistas dosificadores de la red de salud UC-CHRISTUS, La razón de controles fuera de rango se calcula con la cantidad total de controles fuera de rango/ total de controles (según rango de anticoagulación establecido particular para cada paciente [rango de INR]). Estos datos se separaron en grupos según el fármaco que cada paciente utilizó durante su tratamiento. Posteriormente, se calculó una razón de probabilidades (Odds Ratio, OR), comparado las proporciones de controles fuera de rango/ total de controles entre cada uno de los fármacos bioequivalentes del acenocumarol (Acebrón ${ }^{\circledast}$, $\mathrm{Coarol}^{\circledast}$ e Isquelium ${ }^{\circledast}$ ) con el fármaco de referencia $\left(\mathrm{Neo}^{-S i n t r o m}{ }^{\circledast}\right.$ ) y los fármacos no bioequivalentes del acenocumarol (Acenox ${ }^{\oplus}$ acenocumarol genérico) con el fármaco de referencia. Además, debido a que por el alto costo de los medicamentos 
muchos pacientes lo obtienen encargándolo al extranjero, se comparó el fármaco Sintrom ${ }^{\circledast}$ que es producido por el mismo laboratorio farmacéutico que Neo-Sintrom ${ }^{\oplus}$. En el caso de la warfarina, debido a que no está considerado ningún fármaco como de referencia por el Instituto de Salud Pública de Chile (ISP) se utilizó como fármaco de comparación Coumadin ${ }^{\circledR}$ debido a que posee el registro más antiguo, además que el mayor número de pacientes fueron tratados con este fármaco y se comparó con el bioequivalente Cavamed ${ }^{\circledR}$. Finalmente se comparó de forma global el OR de controles fuera de rango entre el acenocumarol y la warfarina; dado que la proporción de controles de fármacos no bioequivalentes del acenocumarol es sustancialmente menor a los bioequivalentes, se incluyeron en este análisis global ya que un análisis independiente restaría poder estadístico. Debido a que existen varios médicos dosificadores que intervinieron en la realización de los controles, se realizó un análisis aislado para cada uno de ellos y posteriormente un análisis global para cada uno de los grupos (random effects). En ciertos casos, algunos médicos no registraron prescripciones de ciertos fármacos, por lo que fueron excluidos en el análisis particular de ese grupo. Los gráficos y cálculos se realizaron utilizando los softwares GraphPad Prism 8.3 (GraphPad Software, San Diego, CA, USA) y MedCalc Statistical Software 16.4.3 (MedCalc Software bv, Ostend, Belgium). El protocolo de investigación y recolección de datos fue aprobado por el comité de ética de la Facultad de Medicina de la Pontificia Universidad Católica de Chile.

\section{Resultados}

Comparación del fármaco de referencia con los fármacos bioequivalentes del acenocumarol (Figura 1A). Se observó una proporción significativamente menor de controles fuera de rango en el grupo de pacientes tratados con Acebrón ${ }^{\otimes}(5.459 / 10.950)$ en comparación a los tratados con Neo-Sintrom ${ }^{\otimes}$ (18.344/33.560) (OR 0,812 IC $_{95}$ 0,756-0,872; $\mathrm{P}<0,001)$. No se observó diferencia entre Coarol ${ }^{\oplus}$ (3.888/7.298) y Neo-Sintrom ${ }^{\varpi}(18.344 / 33.560)$ (OR 0,969 IC $_{95}$ 0,889-1,056; $\mathrm{P}=0,475$ ). No se observó diferencia entre Isquelium ${ }^{\circledast}(1.328 / 2.442)$ y Neo-Sintrom ${ }^{\varpi}(18.344 / 33.560)$ (OR 0,974 $\mathrm{IC}_{95}$ 0,837-1,132; $\left.\mathrm{P}=0.729\right)$. No se observó diferencia entre pacientes tratados con Sintrom ${ }^{\oplus}$ $(1.524 / 2.833)$ y Neo-Sintrom ${ }^{\circledast}(17.192 / 31.480)$ (OR 0,963 $\left.\mathrm{IC}_{95} 0,810-1,146 ; \mathrm{P}=0,673\right)$.

Comparación del fármaco de referencia con los fármacos no bioequivalentes del acenocumarol (Figura 1B). Se observó una proporción significativamente mayor de controles fuera de rango en el grupo de pacientes tratados con acenocumarol genérico (668/1.015) en comparación a los tratados con Neo-Sintrom ${ }^{\circledast}(17.192 / 31.480)$ (OR 1,561 IC ${ }_{95}$ $1,367-1,781 ; \mathrm{P}<0,001)$. Se observó una propor-

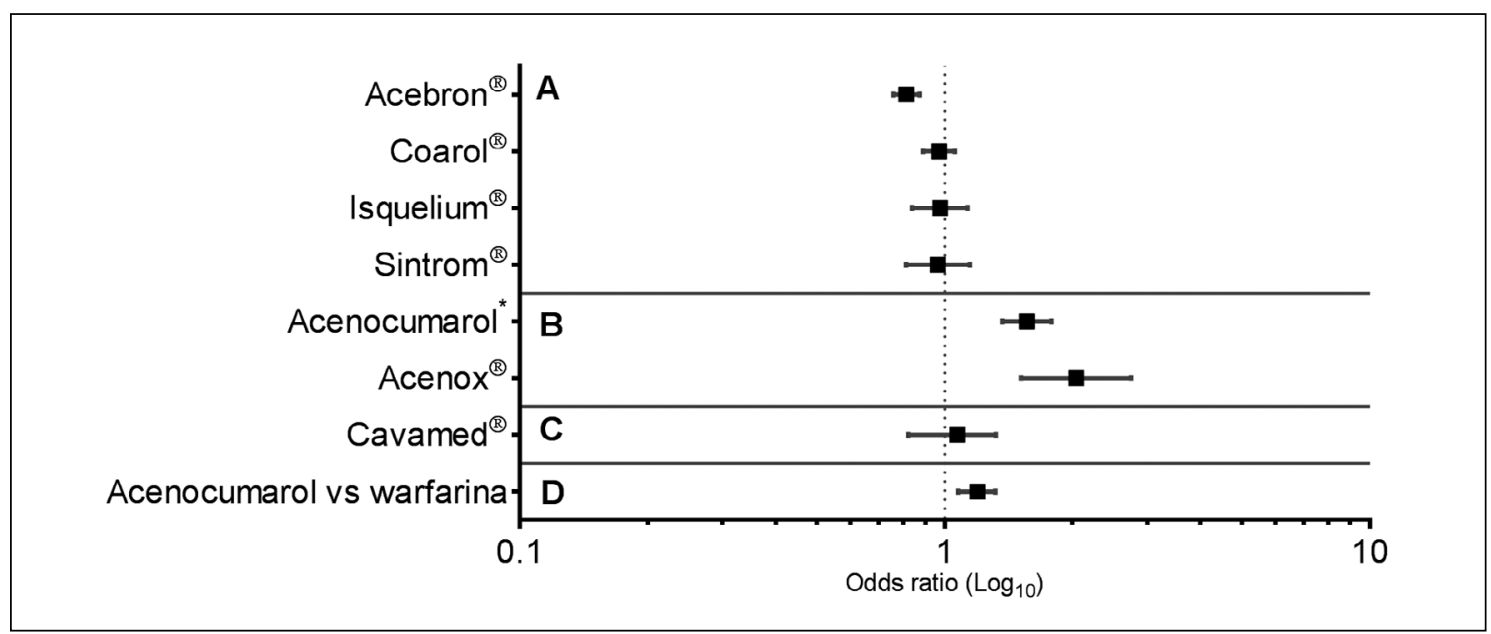

Figura 1. $\mathrm{OR}\left( \pm \mathrm{IC}_{95}\right)$ de las comparaciones de los bioequivalentes $(\mathbf{A})$ y genéricos $(\mathbf{B})$ con el fármaco de referencia del acenocumarol. Comparación de las marcas de warfarina, tomando como referencia Coumadin ${ }^{\circledR}(\mathbf{C})$. Comparación global entre acenocumarol y warfarina. *Acenocumarol genérico. 
ción significativamente mayor de controles fuera de rango en el grupo de pacientes tratados con Acenox $^{\oplus}(162 / 226)$ en comparación a los tratados con Neo-Sintrom ${ }^{\circledast}(12.945 / 23.736)$ (OR 2,037 IC I5 $_{9}$ 1,513-2,744; $\mathrm{P}<0,001)$.Comparación de fármacos bioequivalentes de la warfarina (Figura 1C). No se observó diferencia entre Cavamed ${ }^{\circledast}(706 / 1.375)$ y Coumadin ${ }^{\varpi}(4.768 / 9.623)$ (OR 1,050 $\mathrm{IC}_{95}$ 0,831$1,328 ; \mathrm{P}=0,680)$.

Comparación global de acenocumarol y warfarina (Figura 1D). Se observó una proporción significativamente mayor de controles fuera de rango en el grupo de pacientes tratados con acenocumarol (29.130/54.357) en comparación a los tratados con warfarina (5.474/10.998) (OR 1,191 $\mathrm{IC}_{95}$ 1,077-1,317; $\left.\mathrm{P}=0,001\right)$.

\section{Discusión}

Los medicamentos genéricos constituyen un componente importante en los sistemas de salud, por ejemplo representan aproximadamente $90 \%$ de todas las recetas prescritas en los Estados Unidos de América ${ }^{17}$, país donde los medicamentos genéricos son aprobados por la Food and Drugs Administration (FDA) basada en la demostración de equivalencia terapéutica, incluida la bioequivalencia con el producto de la marca. Específicamente, los intervalos de confianza de $90 \%$ de los parámetros farmacocinéticos clave que requiere que la absorción del medicamento genérico en plasma sea dentro de $80 \%$ y $125 \%$ de estos parámetros para el medicamento evaluado ${ }^{18}$.

Los fármacos de índice terapéutico estrecho (ITE), se definen como productos para los cuales hay menos de dos veces la diferencia entre la concentración tóxica mínima y la concentración efectiva mínima ${ }^{19}$, y han sido objeto de mayor escrutinio ${ }^{20}$. Aunque los estándares de bioequivalencia son algo más estrictos para los productos ITE, lo que requiere el uso de un enfoque de escala promedio de referencia para escalar a la variabilidad del producto de referencia y una comparación del producto genérico y de marca dentro del tema variabilidades ${ }^{21}$.

Como medicamento de tipo ITE, se ha postulado durante mucho tiempo que incluso pequeños cambios en la concentración plasmática de warfarina podrían conducir a cambios importantes en la respuesta farmacodinámica, lo que podría com- prometer la relación riesgo-beneficio ${ }^{22}$. Sin embargo, estudios internacionales, incluidos ensayos controlados aleatorios y estudios observacionales, han proporcionado resultados muy consistentes con respecto a la seguridad y la efectividad de los productos de warfarina genéricos comparados con los de marca ${ }^{23,24}$. En ensayos aleatorios, no se observó diferencias significativas en la media del INR que se observa después de cambiar entre productos de warfarina de marca y genéricos ${ }^{22,25-27}$. En cuanto a desenlaces clínicos, un estudio observacional que involucró 33.645 pacientes de 65 años o más que iniciaron warfarina, no se observó diferencia sustanciales en los desenlaces clínicos, incluyendo accidente cerebrovascular o tromboembolismo venoso, hemorragia mayor y mortalidad por todas las causas, entre aquellos que iniciaron warfarina genérica versus marca ${ }^{28}$. Así mismo, una gran investigación ecológica de Canadá no encontró diferencias significativas en las tasas de hospitalizaciones por hemorragia o tromboembolismo cerebral antes y después de la implementación de la política genérica de sustitución de warfarina ${ }^{29}$. En un reciente estudio observacional realizado en una cohorte danesa, se informó un riesgo similar como punto final de seguridad, anticoagulación excesiva que abarca un aumento de INR o hemorragia mayor, pero se observó un mayor riesgo de eventos tromboembólicos (RR 2,4 IC 95\% 1,7-3,2) entre los usuarios de genéricos en comparación con los usuarios de warfarina de marca ${ }^{30}$.

Los resultados planteados en la revisión sistemática realizada por Dentali et al. ${ }^{23}$, sugieren que los productos genéricos de warfarina que cumplen con los estándares de bioequivalencia de la FDA son probablemente tan seguros y efectivos como los productos de marca y que los pacientes pueden ser tratados de forma segura con warfarina genérica. Sin embargo, es razonable una supervisión más estrecha y frecuente al cambiar de marca, ya que pueden observarse variaciones en la respuesta individual y sería prudente que los pacientes cambien con poca frecuencia entre las presentaciones comerciales de warfarina, lo que permite mantener una dosis estable de warfarina. Debido a que en países anglosajones es la warfarina el fármaco AVK mayoritariamente usado, la información y comparación entre medicamentos de marca, bioequivalentes y genéricos del acenocumarol es limitada. En un estudio realizado en 
Chile Sasso et al. ${ }^{31}$ compararon los parámetros farmacocinéticos de Acebron ${ }^{\oplus}$ y Neo-Sintrom ${ }^{\oplus}$ en pacientes voluntarios sanos, no encontrando diferencias significativas entre ambas formulaciones en los valores del área bajo la curva (AUC) o $\mathrm{C}_{\mathrm{max}}$, sugiriendo que $4 \mathrm{mg}$ de Neo-Sintrom ${ }^{\oplus}$ y Acebron son bioequivalentes e intercambiables. En nuestra investigación, al igual que el Acebron ${ }^{\oplus}$, los otros fármacos considerados bioequivalentes del acenocumarol, muestran proporciones de controles fuera de rango terapéutico estadísticamente similares al Neo-Sintrom ${ }^{\oplus}$, caso contrario con los fármacos no registrados como bioequivalentes, donde las proporciones de controles fuera de rango terapéutico fueron estadísticamente mayores respecto al Neo-Sintrom ${ }^{\oplus}$. A pesar de que la evidencia de comparación entre marcas es más limitada en el caso del acenocumarol debido al menor número de lugares en el mundo donde se utiliza como $\mathrm{AVK}$, es posible que las precauciones inferidas para el caso de la warfarina sean extrapolables a los pacientes en los cuales se utiliza para su control.

En cuanto a la eficacia del tratamiento anticoagulante con acenocumarol, en nuestro estudio las proporciones de controles fuera de rango terapéutico fueron estadísticamente mayores en pacientes tratados con acenocumarol (OR 1,191 $\mathrm{IC}_{95}$ 1.077-1.317), concordante con Pattacini et al. ${ }^{32}$ donde determinaron en grupos independientes, que la calidad del tratamiento general fue significativamente mejor en los pacientes tratados con warfarina que con acenocumarol (72\% y $67 \%$ de controles en rango terapéutico respectivamente). Por su parte, en la investigación realizada por Berini et al. ${ }^{33}$, los pacientes tratados con acenocumarol por un año, comparados con los tratados con warfarina, exhibieron mayor riesgo de presentar un INR $\geq 6$ ( 0,3 y 0,07 visitas/ paciente/año respectivamente), pero sin diferencia estadística observada en la estabilidad terapéutica entre ambos fármacos.

Es importante mencionar que la mayor limitante metodológica de este estudio es su origen transversal, que imposibilita contrastar los resultados con aquellos de tipo longitudinal. A esto se suma el desconocimiento de desenlaces clínicos, la variabilidad intra-paciente y el TRT, datos que precisan de forma más fidedigna la eficacia del tratamiento anticoagulante a diferencia del análisis individualizado para cada control. Así mismo, en cuanto al poder estadístico, es necesario destacar que la muestra de controles de pacientes que utilizaron formulaciones de acenocumarol bioequivalentes permitió aplicar las pruebas estadísticas tradicionales sin problemática, pero la proporción respecto a los controles de pacientes que utilizaron acenocumarol de tipo no bioequivalente, fue considerablemente menor; además es necesario tener en cuenta el nivel de heterogeneidad interdosificador determinado en cada grupo de comparación (en especial en la comparación del fármaco de referencia con Isquelium ${ }^{\circledast}$ y Sintrom ${ }^{\oplus}$, así como en la comparación entre marcas de warfarina y el contraste global entre acenocumarol y warfarina, donde la heterogeneidad fue alta $(>50 \%)$. En general, aproximadamente una cuarta parte de los análisis de estudios poseen valores de heterogeneidad superiores a $50 \%{ }^{34}$ por lo que es necesario tener en cuenta que su cuantificación es solo un componente de análisis de una investigación ${ }^{35}$, siendo más importante la perspectiva centrada en aspectos metodológicos, considerando las implicaciones clínicas, dirección del efecto medido e intervalo de confianza.

Agradecimientos: Los autores agradecen al Dr. Oslando Padilla del Departamento de Salud Pública de la Facultad de Medicina de la Pontificia Universidad Católica de Chile, por la asesoría estadística otorgada.

\section{Referencias}

1. Nieto E, Suárez M, Roco Á, Rubilar JC, Tamayo F, Rojo $\mathrm{M}$, et al. Anticoagulation Management With Coumarinic Drugs in Chilean Patients. Clin Appl Thromb 2019; 25. Disponible en: https://journals.sagepub.com/ doi/10.1177/1076029619834342 [Consultado el 11 de noviembre de 2019].

2. DEIS, Departamento de Estadísticas e Información en Salud M de S-C. No Title [Internet]. 2018. Disponible en: http://www.deis.cl/wp-content/uploads/2015/05/ Serie-defunciones-y-mortalidad-causas-regiones.-Chile-1997-2012.xlsx [Consultado el 11 de noviembre de 2019].

3. Rieder MJ, Reiner AP, Gage BF, Nickerson DA, Eby CS, McLeod HL, et al. Effect of VKORC1 haplotypes on transcriptional regulation and warfarin dose. $\mathrm{N}$ Engl J Med 2005; 352 (22): 2285-93. Disponible en: https:// www.nejm.org/ [Consultado el 22 de octubre 2019].

4. Predisposición genética al sangrado durante el tratamiento con anticoagulantes orales. Disponible en: 
http://scielo.isciii.es/scielo.php?script=sci_arttext\&pi$\mathrm{d}=$ S1137-66272008000500005 [Consultado el $21 \mathrm{de}$ enero de 2020].

5. Benavides F, Grossman N, Poggi H, Nieto E, Bertrán A, Araos D, et al. Efecto de las variantes de vkorcl y cyp2c9 sobre la dosis de anticoagulantes orales en individuos chilenos. Rev Med Chile 2015; 143 (11): 1369-76. Disponible en: https://scielo.conicyt.cl/scielo.php?scrip$\mathrm{t}=$ sci_abstract\&pid=S0034-98872015001100001\&ln$\mathrm{g}=$ es\&nrm=iso [Consultado el 22 de octubre de 2019].

6. Schwarz UI, Ritchie MD, Bradford Y, Li C, Dudek SM, Frye-Anderson A, et al. Genetic determinants of response to warfarin during initial anticoagulation. $\mathrm{N}$ Engl J Med. 2008; 358 (10): 999-1008. Disponible en: https:// www.nejm.org/doi/full/10.1056/NEJMoa0708078 [Consultado el 24 de febrero de 2020].

7. Jacobs LG. Warfarin pharmacology, clinical management, and evaluation of hemorrhagic risk for the elderly. Vol. 22, Clinics in Geriatric Medicine. W.B. Saunders; 2006. p. 17-32. Disponible en: https://www.sciencedirect.com/science/article/abs/pii/S0749069005000674?via\%3Dihub [Consultado el 24 de febrero de 2020].

8. Aithal GP, Day CP, Kesteven PJL, Daly AK. Association of polymorphisms in the cytochrome P450 CYP2C9 with warfarin dose requirement and risk of bleeding complications. Lancet. 1999; 353 (9154): 717-9. Disponible en: https://www.thelancet.com/journals/lancet/ article/PIIS0140-6736(05)77184-1/fulltext [Consultado el 17 de diciembre de 2019].

9. Xie HG, Prasad HC, Kim RB, Stein CM. CYP2C9 allelic variants: Ethnic distribution and functional significance. Adv Drug Deliv Rev. 2002; 54 (10): 1257-70. Disponible de: https://www.sciencedirect.com/science/article/pii/ S0169409X02000765?via\%3Dihub [consultado el 11 de octubre de 2029].

10. Rosendaal FR, Cannegieter SC, Van der Meer FJM, Briet E. A method to determine the optimal intensity of oral anticoagulant therapy. Thromb Haemost 1993; 69 (3): 236-9. Disponible de: openacces.leidenuniv.nl [consultado el 11 de octubre de 2019].

11. Haas S, Cate H Ten, Accetta G, Angchaisuksiri P, Bassand JP, John Camm A, et al. Quality of vitamin k antagonist control and 1-year outcomes in patients with atrial fibrillation: A global perspective from the GARFIELD-AF registry. PLoS One. 2016; 11 (10). Disponible de: https://journals.plos.org/plosone/article?id=10.1371/ journal.pone.0164076 [Consultado el 21 de enero de 2020].

12. Overview | Atrial fibrillation: management | Guidance | NICE. Disponible en: https://www.nice.org.uk/guidance/cg180 [Consulado el 21 de enero de 2020].
13. Camm AJ, Lip GYH, De Caterina R, Savelieva I, Atar D, Hohnloser SH, et al. 2012 focused update of the ESC Guidelines for the management of atrial: Fibrillation An update of the 2010 ESC Guidelines for the management of atrial fibrillation: Developed with the special contribution of the European Heart Rhythm Association. Vol. 14, Europace. Oxford University Press; 2012. p. 1385-413. Disponible en: https://academic.oup.com/ eurheartj/article/33/21/2719/493051 [Consultado el 21 de enero de 2020].

14. Ogawa S, Aonuma K, Tse H-F, Huang D, Huang J-L, Kalman J, et al. The APHRS's 2013 statement on antithrombotic therapy of patients with nonvalvular atrial fibrillation. J Arrhythmia. 2013; 29 (3): 190-200. Dispobible de: http://doi.wiley.com/10.1016/j.joa.2013.03.002 [Consultado el 21 de enero de 2020].

15. Borobia AM, Lubomirov R, Ramírez E, Lorenzo A, Campos A, Muñoz-Romo R, et al. An acenocoumarol dosing algorithm using clinical and pharmacogenetic data in Spanish patients with thromboembolic disease. PLoS One 2012; 7 (7). Disponible en: https:// journals.plos.org/plosone/article?id=10.1371/journal. pone.0041360 [Consultado el 21 de enero de 2020].

16. Tuteja S, Limdi N. Pharmacogenetics in Cardiovascular Medicine. Curr Genet Med Rep 2016; 4 (3): 119-29. Disponible en: https://link.springer.com/article/10.1007\%2Fs40142-016-0096-z [Consultado el 21 de enero de 2020].

17. Nash DB. The use of medicines in the united states: A detailed review. Am Heal Drug Benefits. 2012; 5 (7). Disponible en: https://www.ncbi.nlm.nih.gov/pmc/ articles/PMC4046483/pdf/ahdb-05-423.pdf [Consultado el 21 de enero de 2020].

18. Fda, Cder, Winbourne. Guidance for Industry Bioequivalence Studies with Pharmacokinetic Endpoints for Drugs Submitted Under an ANDA. Disponible en: http://www.fda.gov/Drugs/GuidanceComplianceRegulatoryInformation/Guidances/default.htm. [Consultado el 21 de enero de 2020].

19. Levy G. What are narrow therapeutic index drugs? Vol. 63, Clinical Pharmacology and Therapeutics. 1998. p. 501-5. Disponible en: https://ascpt.onlinelibrary.wiley. com/doi/10.1016/S0009-9236\%2898\%2990100-X [Consultado el 10 de enero de 2020].

20. Hottinger M, Liang BA. Deficiencies of the FDA in evaluating Generic formulations: Addressing narrow therapeutic index drugs. Am J Law Med 2012; 38 (4): 667-89. Disponible en: https://journals.sagepub.com/ doi/10.1177/009885881203800403 [Consultado el 10 de enero de 2020].

21. Gagne JJ, Choudhry NK, Kesselheim AS, Polinski JM, 
Hutchins D, Matlin OS, et al. Comparative effectiveness of generic and brand-name statins on patient outcomes: A cohort study. Ann Intern Med. 2014; 161 (6): 400-7. Disponible en: https://annals.org/aim/article-abstract/1905128/comparative-effectiveness-generic-brand-name-statins-patient-outcomes-cohort-study [Consultado el 10 de enero de 2020].

22. Weibert RT, Yeager BF, Wittkowsky AK, Bussey HI, Wilson DB, Godwin JE, et al. A randomized, crossover comparison of warfarin products in the treatment of chronic atrial fibrillation. Ann Pharmacother 2000; 34 (9): 981-8. Disponible en: https://journals.sagepub.com/ doi/abs/10.1345/aph.10068 [Consultado el 10 de enero de 2020].

23. Dentali F, Donadini MP, Clark N, Crowther MA, Garcia D, Hylek E, et al. Brand name versus generic warfarin: a systematic review of the literature. Pharmacotherapy 2011; 31 (4): 386-93. Disponible en: https:// accpjournals.onlinelibrary.wiley.com/doi/abs/10.1592/ phco.31.4.386 [Consultado el 15 de enero de 2020].

24. Kesselheim AS, Misono AS, Lee JL, Stedman MR, Brookhart MA, Choudhry NK, et al. Clinical equivalence of generic and brand-name drugs used in cardiovascular disease: A systematic review and meta-analysis. Vol. 300, JAMA - Journal of the American Medical Association. 2008. p. 2514-26. Disponible en: https://jamanetwork.com/journals/jama/article-abstract/1028758 [Consultado el 15 de enero de 2020].

25. Lee HL, Kan CD, Yang YJ. Efficacy and tolerability of the switch from a branded toa generic garfarin sodium product: An observer-blinded, randomized, crossover study. Clin Ther 2005; 27 (3): 309-19. Disponible en: https://linkinghub.elsevier.com/retrieve/pii/S01492918(05)00047-0 [Consultado el 10 de enero de 2020].

26. Neutel JM, Smith DH. A randomized crossover study to compare the efficacy and tolerability of Barr warfarin sodium to the currently available Coumadin. Cardiovasc Rev Rep .1998. 19: 49-52, 55-9.

27. Pereira JA, Holbrook AM, Dolovich L, Goldsmith C, Thabane L, Douketis JD, et al. Are brand-name and generic warfarin interchangeable? Multiple N-of-1 randomized, crossover trials. Ann Pharmacother 2005; 39 (7-8): 118893. Disponible en: https://journals.sagepub.com/doi/ full/10.1345/aph.1G003?url_ver=Z39.88-2003\&rfr_id=ori:rid:crossref.org\&rfr_dat $=$ cr_pub $\% 20 \% 200$ pubmed [Consultado el 10 de enero de 2020].

28. Desai RJ, Gopalakrishnan C, Dejene S, Sarpatwari AS, Levin R, Dutcher SK, et al. Comparative outcomes of treatment initiation with brand versus generic warfarin in older patients. Clin Pharmacol Ther 2019; cpt.1743. Disponible en: https://ascpt.onlinelibrary.wiley.com/ doi/abs/10.1002/cpt.1743 [Consultado el 11 de enero de 2020].

29. Paterson JM, Mamdani M, Juurlink DN, Naglie G, Laupacis A, Stukel TA. Clinical consequences of generic warfarin substitution: an ecological study. JAMA 2006; 296 (16): 1969-72. Disponible en: https://jamanetwork. com/journals/jama/article-abstract/203749 [Consultado el 10 de enero de 2020].

30. Hellfritzsch M, Rathe J, Stage TB, Thirstrup S, Grove EL, Damkier P, et al. Generic switching of warfarin and risk of excessive anticoagulation: A Danish nationwide cohort study. Pharmacoepidemiol Drug Saf 2016; 25 (3): 336-43. Disponible en: https://onlinelibrary.wiley.com/ doi/abs/10.1002/pds.3942 [Consultado el 15 de enero de 2020].

31. Sasso J, Carmona P, Quiñones L, Ortiz M, Tamayo E, Varela N, et al. Bioequivalence of acenocoumarol in chilean volunteers: An open, randomized, double-blind, single-dose, 2-period, and 2-sequence crossover study for 2 oral formulations. Arzneimittel-Forschung/ Drug Res 2012; 62 (8): 395-9. Disponible en: https:// www.thieme-connect.de/products/ejournals/abstract/10.1055/s-0032-1316290 [Consultado el 09 de enero de 2020].

32. Pattacini C, Manotti C, Pini M, Quintavalla R, Dettori AG. A comparative study on the quality of oral anticoagulant therapy (Warfarin versus acenocoumarol). Thromb Haemost 1994; 71 (2): 188-91. Disponible en: https://www.thrombosisresearch.com/article/0049-3848(93)90295-Y/fulltext [Consultado el 09 de enero de 2020].

33. Oliva Berini E, Galán Alvarez P, Pacheco Onrubia AM. Comparación de la calidad y el riesgo hemorrágico del tratamiento anticoagulante oral con acenocumarol frente a warfarina. Med Clin (Barc) 2008; 131 (3): 96-7. Disponible en: https://linkinghub.elsevier.com/retrieve/ pii/S0025775308716142 [Consultado el 23 de enero de 2020].

34. Sterne JAC, Bradburn MJ EM. Systematic reviews in health care: meta-analysis in context. 2nd ed. London: BMJ Publications; 2001. 347-69 p.35. Higgins JPT, Thompson SG, Deeks JJ, Altman DG. Measuring inconsistency in meta-analyses. Vol. 327, British Medical Journal. 2003. p. 557-60. Disponible en: https://www. bmj.com/content/327/7414/557 [Consultado el: 10 de marzo de 2020].

35. Higgins JPT, Thompson SG, Deeks JJ, Altman DG. Measuring inconsistency in meta-analyses. BMJ 2003; 327 (7414): 557-60. Disponible en: https://www.bmj.com/ content/327/7414/557 [Consultado el 10 de marzo de 2020]. 